\title{
Schur convexity properties for a class of symmetric functions with applications
}

\section{Wei-Mao Qian ${ }^{\mathrm{a}}$, Yu-Ming Chu ${ }^{\mathrm{b}, *}$}

a School of Distance Education, Huzhou Broadcast and TV University, Huzhou 313000, China.

${ }^{b}$ Department of Mathematics, Huzhou University, Huzhou 313000, China.

\section{Abstract}

In the article, we prove that the symmetric function

$$
F_{n}\left(x_{1}, x_{2}, \cdots, x_{n} ; r\right)=\sum_{1 \leqslant i_{1}<i_{2}<\cdots<i_{r} \leqslant n} \prod_{j=1}^{r}\left(\frac{1+x_{i_{j}}}{1-x_{i_{j}}}\right)^{1 / r}
$$

is Schur convex, Schur multiplicatively convex and Schur harmonic convex on $[0,1)^{n}$, and establish several new analytic inequalities by use of the theory of majorization, where $r \in\{1,2, \cdots, n\}$ and $i_{1}, i_{2}, \cdots i_{n}$ are integers.

Keywords: Schur convex, Schur multiplicatively convex, Schur harmonic convex, symmetric function. 2010 MSC: 05E05, 26B25.

\section{Introduction}

Throughout this paper, we use $\mathbb{R}^{n}$ to denote the $n$-dimensional Euclidean space $(n \geqslant 2)$, and $\mathbb{R}_{+}^{n}=$ $\left\{\left(x_{1}, x_{2}, \cdots, x_{n}\right): x_{i}>0, i=1,2, \cdots, n\right\}$. In particular, we use $\mathbb{R}$ to denote $\mathbb{R}^{1}$.

For the sake of convenience, we use the following notation system.

Let $x=\left(x_{1}, x_{2}, \cdots, x_{n}\right), y=\left(y_{1}, y_{2}, \cdots, y_{n}\right) \in \mathbb{R}_{+}^{n}$ and $\alpha \in \mathbb{R}$. Then we denote by

$$
\begin{aligned}
x \pm y & =\left(x_{1} \pm y_{1}, x_{2} \pm y_{2}, \cdots, x_{n} \pm y_{n}\right), x y=\left(x_{1} y_{1}, x_{2} y_{2}, \cdots, x_{n} y_{n}\right) \\
\alpha x & =\left(\alpha x_{1}, \alpha x_{2}, \cdots, \alpha x_{n}\right), e^{x}=\left(e^{x_{1}}, e^{x_{2}}, \cdots, e^{x_{n}}\right), \\
\alpha \pm x & =\left(\alpha \pm x_{1}, \alpha \pm x_{2}, \cdots, \alpha \pm x_{n}\right), x^{\alpha}=\left(x_{1}^{\alpha}, x_{2}^{\alpha}, \cdots, x_{n}^{\alpha}\right) \\
\log x & =\left(\log x_{1}, \log x_{2}, \cdots, \log x_{n}\right), \frac{1}{x}=\left(\frac{1}{x_{1}}, \frac{1}{x_{2}}, \cdots, \frac{1}{x_{n}}\right) \\
A_{n}(x) & =\frac{1}{n} \sum_{i=1}^{n} x_{i}, G_{n}(x)=\left(\prod_{i=1}^{n} x_{i}\right)^{\frac{1}{n}} .
\end{aligned}
$$

*Corresponding author

Email addresses: qwm661977@126. com (Wei-Mao Qian), chuyuming2005@126. com (Yu-Ming Chu)

doi: $10.22436 /$ jnsa.011.06.10

Received: 2016-01-24 Revised: 2018-03-27 Accepted: 2018-03-28 
Definition 1.1. A real-valued function $F$ on $E \subseteq \mathbb{R}^{n}$ is said to be Schur convex if

$$
F\left(x_{1}, x_{2}, \cdots, x_{n}\right) \leqslant F\left(y_{1}, y_{2}, \cdots, y_{n}\right)
$$

for each pair of $n$-tuples $x=\left(x_{1}, \cdots, x_{n}\right), y=\left(y_{1}, \cdots, y_{n}\right) \in E$ such that $x \prec y$, i.e.,

$$
\sum_{i=1}^{k} x_{[i]} \leqslant \sum_{i=1}^{k} y_{[i]}, \quad k=1,2, \cdots, n-1
$$

and

$$
\sum_{i=1}^{n} x_{[i]}=\sum_{i=1}^{n} y_{[i]}
$$

where $x_{[i]}$ denotes the $i$ th largest component of $x$. $F$ is said to be Schur concave if $-F$ is Schur convex.

Definition 1.2. A real-valued function $F$ on $E \subseteq \mathbb{R}_{+}^{n}$ is said to be Schur multiplicatively convex if

$$
F\left(x_{1}, x_{2}, \cdots, x_{n}\right) \leqslant F\left(y_{1}, y_{2}, \cdots, y_{n}\right)
$$

for each pair of $n$-tuples $x=\left(x_{1}, x_{2}, \cdots, x_{n}\right), y=\left(y_{1}, y_{2}, \cdots, y_{n}\right) \in E$ such that $\log x \prec \log y$. $F$ is said to be Schur multiplicatively concave if $\frac{1}{\mathrm{~F}}$ is Schur multiplicatively convex.

Definition 1.3. A real-valued function $F$ on $E \subseteq \mathbb{R}_{+}^{n}$ is said to be Schur harmonic convex (concave) if

$$
F\left(x_{1}, x_{2}, \cdots, x_{n}\right) \leqslant(\geqslant) F\left(y_{1}, y_{2}, \cdots, y_{n}\right)
$$

for each pair of $n$-tuples $x=\left(x_{1}, x_{2}, \cdots, x_{n}\right), y=\left(y_{1}, y_{2}, \cdots, y_{n}\right) \in E$ such that $\frac{1}{x} \prec \frac{1}{y}$.

The Schur convexity was introduced by Schur [18] in 1923. It has many important applications in analytic inequalities [1-4, 12, 15, 21-23, 25-29], extended mean values theory $[7,16,17,19,20]$ and other related fields. Recently, the Schur multiplicative and harmonic convexity properties were investigated in $[5,6,8,10,11]$.

For $n \geqslant 2, x=\left(x_{1}, x_{2}, \cdots, x_{n}\right) \in[0,1)^{n}$ and $r \in\{1,2, \cdots, n\}$, the symmetric function $F_{n}(x, r)$ is defined by

$$
F_{n}(x, r)=F_{n}\left(x_{1}, x_{2}, \cdots, x_{n} ; r\right)=\sum_{1 \leqslant i_{1}<i_{2}<\cdots<i_{r} \leqslant n} \prod_{j=1}^{r}\left(\frac{1+x_{i_{j}}}{1-x_{i_{j}}}\right)^{1 / r},
$$

where $i_{1}, i_{2}, \cdots, i_{r}$ are integers.

The main purpose of this paper is to discuss the Schur convexity, Schur multiplicative convexity and Schur harmonic convexity of $F_{n}(x, r)$. As applications, we establish several analytic inequalities by use of the theory of majorization.

\section{Lemmas}

In order to prove our main result we need several lemmas, which we present in this section.

Lemma 2.1 ([14]). Let $\mathrm{E} \subseteq \mathbb{R}^{\mathrm{n}}$ be a symmetric convex set with nonempty interior int $\mathrm{E}$ and $\mathrm{f}: \mathrm{E} \rightarrow \mathbb{R}$ be a continuous symmetric function. If $\mathrm{f}$ is differentiable on int $\mathrm{E}$, then $\mathrm{f}$ is Schur convex on $\mathrm{E}$ if and only if

$$
\left(x_{i}-x_{j}\right)\left(\frac{\partial f}{\partial x_{i}}-\frac{\partial f}{\partial x_{j}}\right) \geqslant 0
$$

for all $i, j=1,2, \cdots, n$ and $x=\left(x_{1}, \cdots, x_{n}\right) \in$ intE. $f$ is Schur concave on $E$ if and only if inequality (2.1) is reversed, where $\mathrm{E}$ is a symmetric set means that $\mathrm{x} \in \mathrm{E}$ implies $\mathrm{P} x \in \mathrm{E}$ for any $\mathrm{n} \times \mathrm{n}$ permutation matrix $\mathrm{P}$. 
Remark 2.2. Since $\mathrm{f}$ is symmetric, the Schur's condition in Lemma 2.1, i.e., (2.1) reduces to

$$
\left(x_{1}-x_{2}\right)\left(\frac{\partial f}{\partial x_{1}}-\frac{\partial f}{\partial x_{2}}\right) \geqslant 0
$$

Lemma 2.3 ([13]). Let $\mathrm{E} \subseteq \mathbb{R}_{+}^{n}$ be a symmetric multiplicatively convex set with nonempty interior intE and $\mathrm{f}: \mathrm{E} \rightarrow \mathbb{R}_{+}$be a continuous symmetric function. If $\mathrm{f}$ is differentiable on int $\mathrm{E}$, then $\mathrm{f}$ is Schur multiplicatively convex on $\mathrm{E}$ if and only if

$$
\left(\log x_{1}-\log x_{2}\right)\left(x_{1} \frac{\partial f}{\partial x_{1}}-x_{2} \frac{\partial f}{\partial x_{2}}\right) \geqslant 0
$$

for all $\mathrm{x}=\left(\mathrm{x}_{1}, \mathrm{x}_{2}, \cdots, \mathrm{x}_{\mathrm{n}}\right) \in \mathrm{int} \mathrm{E}$, where $\mathrm{E}$ is a multiplicatively convex set means that $\mathrm{x}^{1 / 2} \mathrm{y}^{1 / 2} \in \mathrm{E}$ whenever $x, y \in E$.

Lemma 2.4 ([5]). Let $\mathrm{E} \subseteq \mathbb{R}_{+}^{n}$ be a symmetric harmonic convex set with nonempty interior int $\mathrm{E}$ and $\mathrm{f}: \mathrm{E} \rightarrow \mathbb{R}_{+}$ be a continuous symmetric function. If $\mathrm{f}$ is differentiable on int $\mathrm{E}$, then $\mathrm{f}$ is Schur harmonic convex on $\mathrm{E}$ if and only if

$$
\left(x_{1}-x_{2}\right)\left(x_{1}^{2} \frac{\partial f}{\partial x_{1}}-x_{2}^{2} \frac{\partial f}{\partial x_{1}}\right) \geqslant 0
$$

for all $\mathrm{x}=\left(\mathrm{x}_{1}, \mathrm{x}_{2}, \cdots, \mathrm{x}_{\mathrm{n}}\right) \in \mathrm{int} \mathrm{E}$, where $\mathrm{E}$ is a harmonic convex set means that $2 \mathrm{xy} /(\mathrm{x}+\mathrm{y}) \in \mathrm{E}$ whenever $x, y \in E$.

Lemma 2.5 ([9]). Let $x=\left(x_{1}, x_{2}, \cdots, x_{n}\right) \in \mathbb{R}_{+}^{n}$ and $\sum_{i=1}^{n} x_{i}=s$. If $c \geqslant s$, then

$$
\frac{c-x}{\frac{n c}{s}-1}=\left(\frac{c-x_{1}}{\frac{n c}{s}-1}, \frac{c-x_{2}}{\frac{n c}{s}-1}, \cdots, \frac{c-x_{n}}{\frac{n c}{s}-1}\right) \prec\left(x_{1}, x_{2}, \cdots, x_{n}\right)=x .
$$

Lemma 2.6 ([9]). Let $x=\left(x_{1}, x_{2}, \cdots, x_{n}\right) \in \mathbb{R}_{+}^{n}$ and $\sum_{i=1}^{n} x_{i}=s$. If $c \geqslant 0$, then

$$
\frac{c+x}{\frac{n c}{s}+1}=\left(\frac{c+x_{1}}{\frac{n c}{s}+1}, \frac{c+x_{2}}{\frac{n c}{s}+1}, \cdots, \frac{c+x_{n}}{\frac{n c}{s}+1}\right) \prec\left(x_{1}, x_{2}, \cdots, x_{n}\right)=x .
$$

Lemma 2.7 ([24]). Let $x=\left(x_{1}, x_{2}, \cdots, x_{n}\right) \in \mathbb{R}_{+}^{n}$ and $\sum_{i=1}^{n} x_{i}=s$. If $0 \leqslant \lambda \leqslant 1$, then

$$
\frac{s-\lambda x}{n-\lambda}=\left(\frac{s-\lambda x_{1}}{n-\lambda}, \frac{s-\lambda x_{2}}{n-\lambda}, \cdots, \frac{s-\lambda x_{n}}{n-\lambda}\right) \prec\left(x_{1}, x_{2}, \cdots, x_{n}\right)=x .
$$

Simple computations lead to Lemma 2.8 and Lemma 2.9 immediately.

Lemma 2.8. Let $x=\left(x_{1}, x_{2}, \cdots, x_{n}\right) \in \mathbb{R}_{+}^{n}$ and $\sum_{i=1}^{n} x_{i}=$ s. If $0 \leqslant \lambda \leqslant 1$, then

$$
\frac{s+\lambda x}{n+\lambda}=\left(\frac{s+\lambda x_{1}}{n+\lambda}, \frac{s+\lambda x_{2}}{n+\lambda}, \cdots, \frac{s+\lambda x_{n}}{n+\lambda}\right) \prec\left(x_{1}, x_{2}, \cdots, x_{n}\right)=x .
$$

Lemma 2.9. The function $x \rightarrow f(x)=(1+x)^{1-1 / p}(1-x)^{1+1 / p}$ is strictly decreasing on $[0,1)$ if $p \geqslant 1$.

\section{Main Results}

Theorem 3.1. Let $\mathrm{n} \geqslant 2$ and $\mathrm{F}_{\mathrm{n}}(\mathrm{x}, \mathrm{r})$ be defined by (1.1). Then the following statements are true:

(1) $F_{n}(x, r)$ is Schur convex on $[0,1)^{n}$;

(2) $F_{n}(x, r)$ is Schur multiplicatively convex on $[0,1)^{n}$;

(3) $F_{n}(x, r)$ is Schur harmonic convex on $[0,1)^{n}$. 
Proof. We clearly see that $F_{n}(x, r)$ is symmetric and has continuous first partial derivatives on $(0,1)^{n}$. By Lemma 2.1, Remark 2.2, Lemma 2.3 and Lemma 2.4, we only need to prove that

$$
\begin{array}{r}
\left(x_{1}-x_{2}\right)\left(\frac{\partial F_{n}(x, r)}{\partial x_{1}}-\frac{\partial F_{n}(x, r)}{\partial x_{2}}\right) \geqslant 0 \\
\left(\log x_{1}-\log x_{2}\right)\left(x_{1} \frac{\partial F_{n}(x, r)}{\partial x_{1}}-x_{2} \frac{\partial F_{n}(x, r)}{\partial x_{2}}\right) \geqslant 0
\end{array}
$$

and

$$
\left(x_{1}-x_{2}\right)\left(x_{1}^{2} \frac{\partial F_{n}(x, r)}{\partial x_{1}}-x_{2}^{2} \frac{\partial F_{n}(x, r)}{\partial x_{1}}\right) \geqslant 0
$$

for all $x=\left(x_{1}, x_{2}, \cdots, x_{n}\right) \in(0,1)^{n}$ and $r \in\{1,2, \cdots, n\}$.

We divide the proof into four cases.

Case 1. $r=1$. Then (1.1) leads to

$$
\begin{gathered}
F_{n}(x, 1)=F_{n}\left(x_{1}, x_{2}, \cdots, x_{n} ; 1\right)=\sum_{i=1}^{n} \frac{1+x_{i}}{1-x_{i}}, \\
\frac{\partial F_{n}(x, 1)}{\partial x_{i}}=\frac{2}{\left(1-x_{i}\right)^{2}}(i=1,2), \\
\left(x_{1}-x_{2}\right)\left(\frac{\partial F_{n}(x, 1)}{\partial x_{1}}-\frac{\partial F_{n}(x, 1)}{\partial x_{2}}\right)=\frac{2\left(x_{1}-x_{2}\right)^{2}\left(2-x_{1}-x_{2}\right)}{\left(1-x_{1}\right)^{2}\left(1-x_{2}\right)^{2}} \geqslant 0, \\
\left(\log x_{1}-\log x_{2}\right)\left(x_{1} \frac{\partial F_{n}(x, 1)}{\partial x_{1}}-x_{2} \frac{\partial F_{n}(x, 1)}{\partial x_{2}}\right)=2\left(\log x_{1}-\log x_{2}\right) \frac{\left(x_{1}-x_{2}\right)\left(1-x_{1} x_{2}\right)}{\left(1-x_{1}\right)^{2}\left(1-x_{2}\right)^{2}} \geqslant 0
\end{gathered}
$$

and

$$
\left(x_{1}-x_{2}\right)\left(x_{1}^{2} \frac{\partial F_{n}(x, 1)}{\partial x_{1}}-x_{2}^{2} \frac{\partial F_{n}(x, 1)}{\partial x_{1}}\right)=2\left(x_{1}-x_{2}\right)^{2} \frac{x_{1}+x_{2}-2 x_{1} x_{2}}{\left(1-x_{1}\right)^{2}\left(1-x_{2}\right)^{2}} \geqslant 0 .
$$

Case 2. $r=n$. Then it follow from (1.1) that

$$
\begin{gathered}
F_{n}(x, n)=F_{n}\left(x_{1}, x_{2}, \cdots, x_{n} ; n\right)=\prod_{i=1}^{n}\left(\frac{1+x_{i}}{1-x_{i}}\right)^{1 / n}, \\
\frac{\partial F_{n}(x, n)}{\partial x_{i}}=\frac{2 F_{n}(x, n)}{n\left(1-x_{i}^{2}\right)}(i=1,2), \\
\left(x_{1}-x_{2}\right)\left(\frac{\partial F_{n}(x, n)}{\partial x_{1}}-\frac{\partial F_{n}(x, n)}{\partial x_{2}}\right)=\frac{2\left(x_{1}-x_{2}\right)^{2}\left(x_{1}+x_{2}\right) F_{n}(x, n)}{n\left(1-x_{1}^{2}\right)\left(1-x_{2}^{2}\right)} \geqslant 0, \\
\left(\log x_{1}-\log x_{2}\right)\left(x_{1} \frac{\partial F_{n}(x, n)}{\partial x_{1}}-x_{2} \frac{\partial F_{n}(x, n)}{\partial x_{2}}\right)=\frac{2\left(x_{1}-x_{2}\right)\left(\log x_{1}-\log x_{2}\right)\left(1+x_{1} x_{2}\right) F_{n}(x, n)}{n\left(1-x_{1}^{2}\right)\left(1-x_{2}^{2}\right)} \geqslant 0
\end{gathered}
$$

and

$$
\left(x_{1}-x_{2}\right)\left(x_{1}^{2} \frac{\partial F_{n}(x, n)}{\partial x_{1}}-x_{2}^{2} \frac{\partial F_{n}(x, n)}{\partial x_{1}}\right)=\frac{2\left(x_{1}-x_{2}\right)^{2}\left(x_{1}+x_{2}\right) F_{n}(x, n)}{n\left(1-x_{1}^{2}\right)\left(1-x_{2}^{2}\right)} \geqslant 0
$$

Case 3. $n \geqslant 3$ and $r=2$. Then from (1.1) and Lemma 2.9 we clearly see that

$$
\begin{aligned}
F_{n}(x, 2) & =F_{n}\left(x_{1}, x_{2}, \cdots, x_{n} ; 2\right) \\
& =\left(\frac{1+x_{1}}{1-x_{1}}\right)^{1 / 2}\left(\frac{1+x_{2}}{1-x_{2}}\right)^{1 / 2}+\left(\frac{1+x_{1}}{1-x_{1}}\right)^{1 / 2} \sum_{3 \leqslant i \leqslant n}\left(\frac{1+x_{i}}{1-x_{i}}\right)^{1 / 2}+F_{n-1}\left(x_{2}, x_{3}, \cdots, x_{n} ; 2\right)
\end{aligned}
$$




$$
\begin{aligned}
& =\left(\frac{1+x_{1}}{1-x_{1}}\right)^{1 / 2}\left(\frac{1+x_{2}}{1-x_{2}}\right)^{1 / 2}+\left(\frac{1+x_{2}}{1-x_{2}}\right)^{1 / 2} \sum_{3 \leqslant i \leqslant n}\left(\frac{1+x_{i}}{1-x_{i}}\right)^{1 / 2}+F_{n-1}\left(x_{1}, x_{3}, \cdots, x_{n} ; 2\right), \\
& \frac{\partial F_{n}(x, 2)}{\partial x_{1}}=\left(\frac{1+x_{2}}{1-x_{2}}\right)^{1 / 2} \frac{\left(1+x_{1}\right)^{-1 / 2}}{\left(1-x_{1}\right)^{3 / 2}}+\frac{\left(1+x_{1}\right)^{-1 / 2}}{\left(1-x_{1}\right)^{3 / 2}} \sum_{3 \leqslant i \leqslant n}\left(\frac{1+x_{i}}{1-x_{i}}\right)^{1 / 2}, \\
& \frac{\partial F_{n}(x, 2)}{\partial x_{2}}=\left(\frac{1+x_{1}}{1-x_{1}}\right)^{1 / 2} \frac{\left(1+x_{2}\right)^{-1 / 2}}{\left(1-x_{2}\right)^{3 / 2}}+\frac{\left(1+x_{2}\right)^{-1 / 2}}{\left(1-x_{2}\right)^{3 / 2}} \sum_{3 \leqslant i \leqslant n}\left(\frac{1+x_{i}}{1-x_{i}}\right)^{1 / 2}, \\
& \left(x_{1}-x_{2}\right)\left(\frac{\partial F_{n}(x, 2)}{\partial x_{1}}-\frac{\partial F_{n}(x, 2)}{\partial x_{2}}\right) \\
& =\frac{\left(x_{1}-x_{2}\right)^{2}\left(x_{1}+x_{2}\right)}{\left(1+x_{1}\right)^{1 / 2}\left(1-x_{1}\right)^{3 / 2}\left(1+x_{2}\right)^{1 / 2}\left(1-x_{2}\right)^{3 / 2}}+\left(x_{1}-x_{2}\right) \\
& \times\left[\frac{1}{\left(1+x_{1}\right)^{1 / 2}\left(1-x_{1}\right)^{3 / 2}}-\frac{1}{\left(1+x_{2}\right)^{1 / 2}\left(1-x_{2}\right)^{3 / 2}}\right] \sum_{3 \leqslant i \leqslant n}\left(\frac{1+x_{i}}{1-x_{i}}\right)^{1 / 2} \geqslant 0, \\
& \left(\log x_{1}-\log x_{2}\right)\left(x_{1} \frac{\partial F_{n}(x, 2)}{\partial x_{1}}-x_{2} \frac{\partial F_{n}(x, 2)}{\partial x_{2}}\right) \\
& =\frac{\left(x_{1}-x_{2}\right)\left(\log x_{1}-\log x_{2}\right)\left(1+x_{1} x_{2}\right)}{\left(1+x_{1}\right)^{1 / 2}\left(1-x_{1}\right)^{3 / 2}\left(1+x_{2}\right)^{1 / 2}\left(1-x_{2}\right)^{3 / 2}}+\left(\log x_{1}-\log x_{2}\right) \\
& \times\left[\frac{x_{1}}{\left(1+x_{1}\right)^{1 / 2}\left(1-x_{1}\right)^{3 / 2}}-\frac{x_{2}}{\left(1+x_{2}\right)^{1 / 2}\left(1-x_{2}\right)^{3 / 2}}\right] \sum_{3 \leqslant i \leqslant n}\left(\frac{1+x_{i}}{1-x_{i}}\right)^{1 / 2} \geqslant 0
\end{aligned}
$$

and

$$
\begin{aligned}
\left(x_{1}-x_{2}\right) & \left(x_{1}^{2} \frac{\partial F_{n}(x, 2)}{\partial x_{1}}-x_{2}^{2} \frac{\partial F_{n}(x, 2)}{\partial x_{2}}\right) \\
= & \frac{\left(x_{1}-x_{2}\right)^{2}\left(x_{1}+x_{2}\right)}{\left(1+x_{1}\right)^{1 / 2}\left(1-x_{1}\right)^{3 / 2}\left(1+x_{2}\right)^{1 / 2}\left(1-x_{2}\right)^{3 / 2}}+\left(x_{1}-x_{2}\right) \\
& \times\left[\frac{x_{1}^{2}}{\left(1+x_{1}\right)^{1 / 2}\left(1-x_{1}\right)^{3 / 2}}-\frac{x_{2}^{2}}{\left(1+x_{2}\right)^{1 / 2}\left(1-x_{2}\right)^{3 / 2}}\right] \sum_{3 \leqslant i \leqslant n}\left(\frac{1+x_{i}}{1-x_{i}}\right)^{1 / 2} \geqslant 0 .
\end{aligned}
$$

Case $4 . n \geqslant 4$ and $3 \leqslant r \leqslant n-1$. Then (1.1) and Lemma 2.9 lead to

$$
\begin{aligned}
F_{n}(x, r)= & \left(\frac{1+x_{1}}{1-x_{1}}\right)^{1 / r}\left(\frac{1+x_{2}}{1-x_{2}}\right)^{1 / r} \sum_{3 \leqslant i_{1}<i_{2}<\cdots<i_{r-2} \leqslant n} \prod_{j=1}^{r-2}\left(\frac{1+x_{i_{j}}}{1-x_{i_{j}}}\right)^{1 / r} \\
& +\left(\frac{1+x_{1}}{1-x_{1}}\right)^{1 / r} \sum_{3 \leqslant i_{1}<i_{2}<\cdots<i_{r-1} \leqslant n} \prod_{j=1}^{r-1}\left(\frac{1+x_{i_{j}}}{1-x_{i_{j}}}\right)^{1 / r}+F_{n-1}\left(x_{2}, x_{3}, \cdots, x_{n} ; r\right) \\
= & \left(\frac{1+x_{1}}{1-x_{1}}\right)^{1 / r}\left(\frac{1+x_{2}}{1-x_{2}}\right)^{1 / r} \sum_{3 \leqslant i_{1}<i_{2}<\cdots<i_{r-2} \leqslant n} \prod_{j=1}^{r-2}\left(\frac{1+x_{i_{j}}}{1-x_{i_{j}}}\right)^{1 / r} \\
& +\left(\frac{1+x_{2}}{1-x_{2}}\right)^{1 / r} \sum_{3 \leqslant i_{1}<i_{2}<\cdots<i_{r-1} \leqslant n} \prod_{j=1}^{r-1}\left(\frac{1+x_{i_{j}}}{1-x_{i_{j}}}\right)^{1 / r}+F_{n-1}\left(x_{1}, x_{3}, \cdots, x_{n} ; r\right),
\end{aligned}
$$




$$
\begin{aligned}
& \frac{\partial F_{n}(x, r)}{\partial x_{1}}=\frac{2\left(1+x_{1}\right)^{1 / r-1}}{r\left(1-x_{1}\right)^{1 / r+1}}\left(\frac{1+x_{2}}{1-x_{2}}\right)^{1 / r} \sum_{3 \leqslant i_{1}<i_{2} \cdots<i_{r-2} \leqslant n} \prod_{j=1}^{r-2}\left(\frac{1+x_{i_{j}}}{1-x_{i_{j}}}\right)^{1 / r} \\
& +\frac{2}{r\left(1+x_{1}\right)^{1-1 / r}\left(1-x_{1}\right)^{1 / r+1}} \sum_{3 \leqslant i_{1}<i_{2} \cdots<i_{r-1} \leqslant n} \prod_{j=1}^{r-1}\left(\frac{1+x_{i_{j}}}{1-x_{i_{j}}}\right)^{1 / r}, \\
& \frac{\partial F_{n}(x, r)}{\partial x_{2}}=\frac{2\left(1+x_{2}\right)^{1 / r-1}}{r\left(1-x_{2}\right)^{1 / r+1}}\left(\frac{1+x_{1}}{1-x_{1}}\right)^{1 / r} \sum_{3 \leqslant i_{1}<i_{2} \cdots<i_{r-2} \leqslant n} \prod_{j=1}^{r-2}\left(\frac{1+x_{i_{j}}}{1-x_{i_{j}}}\right)^{1 / r} \\
& +\frac{2}{r\left(1+x_{2}\right)^{1-1 / r}\left(1-x_{2}\right)^{1 / r+1}} \sum_{3 \leqslant i_{1}<i_{2} \cdots<i_{r-1} \leqslant n} \prod_{j=1}^{r-1}\left(\frac{1+x_{i_{j}}}{1-x_{i_{j}}}\right)^{1 / r}, \\
& \left(x_{1}-x_{2}\right)\left(\frac{\partial F_{n}(x, r)}{\partial x_{1}}-\frac{\partial F_{n}(x, r)}{\partial x_{2}}\right) \\
& =\frac{2\left(x_{1}-x_{2}\right)}{r} \frac{x_{1}^{2}-x_{2}^{2}}{\left(1-x_{1}^{2}\right)\left(1-x_{2}^{2}\right)}\left(\frac{1+x_{1}}{1-x_{1}}\right)^{1 / r}\left(\frac{1+x_{2}}{1-x_{2}}\right)^{1 / r} \sum_{3 \leqslant i_{1}<i_{2} \cdots<i_{r-2} \leqslant n} \prod_{j=1}^{r-2}\left(\frac{1+x_{i_{j}}}{1-x_{i_{j}}}\right)^{1 / r} \\
& +\frac{2\left(x_{1}-x_{2}\right)}{r}\left[\frac{1}{\left(1+x_{1}\right)^{1-1 / r}\left(1-x_{1}\right)^{1 / r+1}}-\frac{1}{\left(1+x_{2}\right)^{1-1 / r}\left(1-x_{2}\right)^{1 / r+1}}\right] \\
& \times \sum_{3 \leqslant i_{1}<i_{2} \cdots<i_{r-1} \leqslant n} \prod_{j=1}^{r-1}\left(\frac{1+x_{i_{j}}}{1-x_{i_{j}}}\right)^{1 / r} \geqslant 0, \\
& \left(\log x_{1}-\log x_{2}\right)\left(x_{1} \frac{\partial F_{n}(x, r)}{\partial x_{1}}-x_{2} \frac{\partial F_{n}(x, r)}{\partial x_{2}}\right) \\
& =\frac{2}{r}\left(\log x_{1}-\log x_{2}\right) \frac{\left(x_{1}-x_{2}\right)\left(1+x_{1} x_{2}\right)}{\left(1-x_{1}^{2}\right)\left(1-x_{2}^{2}\right)}\left(\frac{1+x_{1}}{1-x_{1}}\right)^{1 / r}\left(\frac{1+x_{2}}{1-x_{2}}\right)^{1 / r} \sum_{3 \leqslant i_{1}<i_{2} \cdots<i_{r-2} \leqslant n} \prod_{j=1}^{r-2}\left(\frac{1+x_{i_{j}}}{1-x_{i_{j}}}\right)^{1 / r} \\
& +\frac{2}{r}\left(\log x_{1}-\log x_{2}\right)\left[\frac{x_{1}}{\left(1+x_{1}\right)^{1-1 / r}\left(1-x_{1}\right)^{1 / r+1}}-\frac{x_{2}}{\left(1+x_{2}\right)^{1-1 / r}\left(1-x_{2}\right)^{1 / r+1}}\right] \\
& \times \sum_{3 \leqslant i_{1}<i_{2} \cdots<i_{r-1} \leqslant n} \prod_{j=1}^{r-1}\left(\frac{1+x_{i_{j}}}{1-x_{i_{j}}}\right)^{1 / r} \geqslant 0
\end{aligned}
$$

and

$$
\begin{aligned}
\left(x_{1}-x_{2}\right) & \left(x_{1}^{2} \frac{\partial F_{n}(x, r)}{\partial x_{1}}-x_{2}^{2} \frac{\partial F_{n}(x, r)}{\partial x_{1}}\right) \\
= & \frac{2\left(x_{1}-x_{2}\right)}{r} \frac{x_{1}^{2}-x_{2}^{2}}{\left(1-x_{1}^{2}\right)\left(1-x_{2}^{2}\right)}\left(\frac{1+x_{1}}{1-x_{1}}\right)^{1 / r}\left(\frac{1+x_{2}}{1-x_{2}}\right)^{1 / r} \sum_{3 \leqslant i_{1}<i_{2} \cdots<i_{r-2} \leqslant n} \prod_{j=1}^{r-2}\left(\frac{1+x_{i_{j}}}{1-x_{i_{j}}}\right)^{1 / r} \\
& +\frac{2\left(x_{1}-x_{2}\right)}{r}\left[\frac{x_{2}^{2}}{\left(1+x_{1}\right)^{1-1 / r}\left(1-x_{1}\right)^{1 / r+1}}-\frac{x_{2}^{2}}{\left(1+x_{2}\right)^{1-1 / r}\left(1-x_{2}\right)^{1 / r+1}}\right] \\
& \times \sum_{3 \leqslant i_{1}<i_{2} \cdots<i_{r-1} \leqslant n}^{r-1}\left(\frac{1+x_{i_{j}}}{1 / r}\right)^{1 / r} \geqslant 0 .
\end{aligned}
$$

Therefore, inequality (3.1) follows from (3.4), (3.7), (3.10) and (3.13), inequality (3.2) follows from (3.5), (3.8), (3.11) and (3.14), and inequality (3.3) follows from (3.6), (3.9), (3.12) and (3.15). 


\section{Applications}

In this section, we establish several analytic inequalities by use of Theorem 3.1 and the theory of majorization.

From Theorem 3.1(1) and Lemmas 2.5-2.8, we get Corollary 4.1 immediately.

Corollary 4.1. If $x=\left(x_{1}, x_{2}, \cdots, x_{n}\right) \in[0,1)^{n}$ with $\sum_{i=1}^{n} x_{i}=s, r \in\{1,2, \cdots, n\}$ and $F_{n}(x, r)$ be defined by (1.1), then we have
(1) $F_{n}(x, r) \geqslant F_{n}\left(\frac{c-x}{\frac{n c}{s}-1}, r\right)$ for $c \geqslant s$;
(2) $F_{n}(x, r) \geqslant F_{n}\left(\frac{c+x}{\frac{n c}{s}+1}, r\right)$ for $c \geqslant 0$;
(3) $F_{n}(x, r) \geqslant F_{n}\left(\frac{s-\lambda x}{n-\lambda}, r\right)$ for $0 \leqslant \lambda \leqslant 1$;
(4) $F_{n}(x, r) \geqslant F_{n}\left(\frac{s+\lambda x}{n+\lambda}, r\right)$ for $0 \leqslant \lambda \leqslant 1$.

Let $x=\left(x_{1}, x_{2}, \cdots, x_{n}\right) \in[0,1)^{n}$. Then from (1.1) and Theorem 3.1(1) together with the fact

$$
\left(A_{n}(x), A_{n}(x), \cdots, A_{n}(x)\right) \prec\left(x_{1}, x_{2}, \cdots, x_{n}\right),
$$

we get Corollary 4.2 .

Corollary 4.2. If $x=\left(x_{1}, x_{2}, \cdots, x_{n}\right) \in[0,1)^{n}, r \in\{1,2, \cdots, n\}$ and $F_{n}(x, r)$ be defined by (1.1), then

$$
F_{n}(x, r) \geqslant \frac{n !}{r !(n-r) !} \frac{A_{n}(1+x)}{A_{n}(1-x)}
$$

Let $\mathrm{r}=1$ and $\mathrm{r}=\mathrm{n}$ in Corollary 4.2, respectively. Then we get Corollary 4.3 immediately.

Corollary 4.3. If $x=\left(x_{1}, x_{2}, \cdots, x_{n}\right) \in[0,1)^{n}$, then

$$
A_{n}\left(\frac{1+x}{1-x}\right) \geqslant \frac{A_{n}(1+x)}{A_{n}(1-x)}, \quad G_{n}\left(\frac{1+x}{1-x}\right) \geqslant \frac{G_{n}(1+x)}{G_{n}(1-x)} .
$$

Corollary 4.4. Let $\mathcal{A}=A_{1} A_{2} \cdots A_{n+1}$ be a n-dimensional simplex in $\mathbb{R}^{n}, P$ be an arbitrary point in the interior of $\mathcal{A}, B_{i}$ be the intersection point of straight line $A_{i} P$ and hyperplane $\sum_{i}=A_{1} A_{2} \cdots A_{i-1} A_{i+1} \cdots A_{n+1}(i=$ $1,2, \cdots, \mathrm{n}+1)$ and $\mathrm{r} \in\{1,2, \cdots, \mathrm{n}+1\}$. Then

$$
\begin{array}{r}
\sum_{1 \leqslant i_{1}<i_{2}<\cdots<i_{r} \leqslant n+1} \prod_{j=1}^{r}\left(1+\frac{2 P B_{i_{j}}}{P A_{i_{j}}}\right)^{1 / r} \geqslant \frac{(n+2) !}{n[r !(n+1-r) !]} \\
\sum_{1 \leqslant i_{1}<i_{2}<\cdots<i_{r} \leqslant n+1} \prod_{j=1}^{r}\left(1+\frac{2 P A_{i_{j}}}{P B_{i_{j}}}\right)^{1 / r} \geqslant \frac{(n+1) !(2 n+1)}{r !(n+1-r) !} .
\end{array}
$$

Proof. We clearly see that

$$
\begin{gathered}
\sum_{i=1}^{n+1} \frac{P B_{i}}{A_{i} B_{i}}=1, \quad \sum_{i=1}^{n+1} \frac{P A_{i}}{A_{i} B_{i}}=n, \\
\left(\frac{1}{n+1}, \frac{1}{n+1}, \cdots, \frac{1}{n+1}\right) \prec\left(\frac{P B_{1}}{A_{1} B_{1}}, \frac{P B_{2}}{A_{2} B_{2}}, \cdots, \frac{P B_{n+1}}{A_{n+1} B_{n+1}}\right), \\
\left(\frac{n}{n+1}, \frac{n}{n+1}, \cdots, \frac{n}{n+1}\right) \prec\left(\frac{P A_{1}}{A_{1} B_{1}} \cdot \frac{P A_{2}}{A_{2} B_{2}}, \cdots, \frac{P A_{n+1}}{A_{n+1} B_{n+1}}\right) .
\end{gathered}
$$

Therefore, Corollary 4.4 follows from (1.1), (4.1), (4.2) and Theorem 3.1(1). 
Corollary 4.5. Suppose that $A=\left(a_{i_{j}}\right)_{n \times n}$ is a complex matrix, and $\lambda_{1}, \lambda_{2}, \cdots, \lambda_{n}$ are the eigenvalues of $A$. If $A$ is a positive definite Hermitian matrix, then one has

$$
\begin{gathered}
\sum_{1 \leqslant i_{1}<i_{2}<\cdots<i_{r} \leqslant n} \prod_{j=1}^{r}\left(\frac{\operatorname{tr} A+\lambda_{i_{j}}}{\operatorname{tr} A-\lambda_{i_{j}}}\right)^{1 / r} \geqslant \frac{(n+1) !}{r !(n-r) !(n-1)}, \\
\sum_{1 \leqslant i_{1}<i_{2}<\cdots<i_{r} \leqslant n} \prod_{j=1}^{r}\left(\frac{\operatorname{tr} A+\lambda_{i_{j}}}{\operatorname{tr} A-\lambda_{i_{j}}}\right)^{1 / r} \geqslant \frac{n !}{r !(n-r) !} \frac{\operatorname{tr} A+\sqrt[n]{\operatorname{tr} A-\sqrt[n]{\operatorname{det} A}}}{\sum_{1 \leqslant i_{1}<i_{2}<\cdots<i_{r} \leqslant n}} \prod_{j=1}^{r}\left(1+\frac{2}{\lambda_{i_{j}}}\right)^{1 / r} \geqslant \frac{n !}{r !(n-r) !}\left(1+\frac{2 n}{\operatorname{tr} A}\right) .
\end{gathered}
$$

Proof. We clearly see that

$$
\begin{aligned}
& \left(\frac{1}{n}, \frac{1}{n}, \cdots, \frac{1}{n}\right) \prec\left(\frac{\lambda_{1}}{\operatorname{tr} A}, \frac{\lambda_{2}}{\operatorname{tr} A}, \cdots, \frac{\lambda_{n}}{\operatorname{tr} A}\right), \\
& \log \left(\frac{\sqrt[n]{\operatorname{det} A}}{\operatorname{tr} A}, \frac{\sqrt[n]{\operatorname{det} A}}{\operatorname{tr} A}, \cdots, \frac{\sqrt[n]{\operatorname{det} A}}{\operatorname{tr} A}\right) \prec \log \left(\frac{\lambda_{1}}{\operatorname{tr} A}, \frac{\lambda_{2}}{\operatorname{tr} A}, \cdots, \frac{\lambda_{n}}{\operatorname{tr} A}\right), \\
& \left(\frac{\operatorname{tr}(A+I)}{n}, \frac{\operatorname{tr}(A+I)}{n}, \cdots, \frac{\operatorname{tr}(A+I)}{n}\right) \prec\left(1+\lambda_{1}, 1+\lambda_{2}, \cdots, 1+\lambda_{n}\right), \\
& \frac{\lambda_{i}}{\operatorname{tr} A}, \frac{1}{1+\lambda_{i}}, \frac{\sqrt[n]{\operatorname{det} A}}{\operatorname{tr} A}, \frac{n}{\operatorname{tr}(A+I)} \in(0,1) .
\end{aligned}
$$

Therefore, the first inequality of Corollary 4.5 follows from Theorem 3.1(1), (4.3) and (4.6), the second inequality of Corollary 4.5 follows from Theorem 3.1(2), (4.4) and (4.6), and the last inequality of Corollary 4.5 follows from Theorem 3.1(3), (4.5) and (4.6).

\section{Acknowledgment}

This research was supported by the Natural Science Foundation of China (Grant Nos. 11701176, 11626101, 11601485) and the Science and Technology Research Program of Zhejiang Educational Committee (Grant No. Y201635325).

\section{References}

[1] M. Adil Khan, S. Begum, Y. Khurshid, Y.-M. Chu, Ostrowski type inequalities involving conformable fractional integrals, J. Inequal. Appl., 2018 (2018), 14 pages. 1

[2] M. Adil Khan, Y.-M. Chu, A. Kashuri, R. Liko, Hermite-Hadamard type fractional integral inequalities for $M T_{(\mathrm{r} ; \mathrm{g}, \mathrm{m}, \varphi)^{-}}$ preinvex functions, J. Comput. Anal. Appl., 26 (2019), 1487-1503.

[3] M. Adil Khan, Y.-M. Chu, T. U. Khan, J. Khan, Some new inequalities of Hermite-Hadamard type for s-convex functions with applications, Open Math., 15 (2017), 1414-1430.

[4] J. S. Aujla, F. C. Silva, Weak majorization inequalities and convex functions, Linear Algebra Appl., 369 (2003), $217-233$. 1

[5] Y.-M. Chu, G.-D. Wang, X.-M. Zhang, The Schur multiplicative and harmonic convexities of the complete symmetric function, Math. Nachr., 284 (2011), 653-663. 1, 2.4

[6] Y.-M. Chu, W.-F. Xia, X.-H. Zhang, The Schur concavity, Schur multiplicative and harmonic convexities of the second dual form of the Hamy symmetric function with applications, J. Multivariate Anal., 105 (2012), 412-421. 1

[7] Y.-M. Chu, X.-M. Zhang, Necessary and sufficient conditions such that extended mean values are Schur-convex or Schurconcave, J. Math. Kyoto Univ., 48 (2008), 229-238. 1

[8] Y.-M. Chu, X.-M. Zhang, G. Wang, The schur geometrical convexity of the extended mean values, J. convex Anal., 15 (2008), 707-718. 1

[9] K. Guan, Schur-convexity of the complete symmetric function, Math. Inequal. Appl., 9 (2006), 567-576. 2.5, 2.6 
[10] K. Guan, A class of symmetric functions for multiplicatively convex function, Math. Inequal. Appl., 10 (2007), 745-753. 1

[11] K. Guan, Some properties of a class of symmetric functions, J. Math. Anal. Appl., 336 (2007), 70-80. 1

[12] G. H. Hardy, J. E. Littledwood, G. Pólya, Some simple inequalities satisfied by convex function, Messenger Math., 58 (1929), 145-152. 1

[13] W.-D. Jiang, Some properties of dual form of the Hamy's symmetric function, J. Math. Inequal., 1 (2007), 117-125. 2.3

[14] A. W. Marshall, I. Olkin, Inequalities: Theory of Majorization and Its Applications, Academic Press, New York-London, (1979). 2.1

[15] M. Merkle, Convexity, Schur-convexity and bounds for the gamma function involving the digamma function, Rocky Mountain J. Math., 28 (1998), 1053-1066. 1

[16] F. Qi, A note on Schur-convexity of extended mean values, Rocky Mountain J. Math., 35 (2005), 1787-1793. 1

[17] F. Qi, J. Sándor, S. S. Dragomir, A. Sofo, Notes on the Schur-convexity of the extended mean values, Taiwanese J. Math., 9 (2005), 411-420. 1

[18] I. Schur, Über eine Klasse von Mittelbildungen mit Anwendungen auf die Determinantentheorie, Sitzunsber. Berlin. Math. Ges., 22 (1923), 9-20. 1

[19] H.-N. Shi, Y.-M. Jiang, W.-D. Jiang, Schur-convexity and Schur-geometrically concavity of Gini means, Comput. Math. Appl., 57 (2009), 266-274. 1

[20] H.-N. Shi, S.-H. Wu, F. Qi, An alternative note on the Schur-convexity of the extended mean values, Math. Inequal. Appl., 9 (2006), 219-224. 1

[21] M.-K. Wang, Y.-M. Chu, Refinements of transformation inequalities for zero-balanced hypergeometric functions, Acta Math. Sci. Ser. B Engl. Ed., 37 (2017), 607-622. 1

[22] M.-K. Wang, Y.-M. Chu, Landen inequalities for a class of hypergeometric functions with applications, Math. Inequal. Appl., 21 (2018), 521-537.

[23] M.-K. Wang, S.-L. Qiu, Y.-M. Chu, Infinite series formula for Hübner upper bound function with applications to HerchPfluger distortion function, Math. Inequal. Appl., 21 (2018), 629-648. 1

[24] S. Wu, Generalization and sharpness of the power means inquality and their applications, J. Math. Anal. Appl., 312 (2005), 637-652. 2.7

[25] Z.-H. Yang, Y.-M. Chu, A monotonicity property involving the generalized elliptic integral of the first kind, Math. Inequal. Appl., 20 (2017), 729-735. 1

[26] Z.-H. Yang, W.-M. Qian, Y.-M. Chu, W. Zhang, On approximating the arithmetic-geometric mean and complete elliptic integral of the first kind, J. Math. Anal. Appl., 462 (2018), 1714-1726.

[27] Z.-H. Yang, W.-M. Qian, Y.-M. Chu, W. Zhang, On approximating the error function, Math. Inequal. Appl., 21 (2018), 469-479.

[28] Z.-H. Yang, W. Zhang, Y.-M. Chu, Sharp Gautschi inequality for parameter $0<p<1$ with applications, Math. Inequal. Appl., 20 (2017), 1107-1120.

[29] X.-M. Zhang, Schur-convex functions and isoperimetric inequalities, Proc. Amer. Math. Soc., 126 (1998), 461-470. 1 